\title{
OCHRATOXIN A KINETICS: A REVIEW OF ANALYTICAL METHODS AND \\ STUDIES IN RAT MODEL
}

\author{
Vettorazzi $\mathbf{A}^{\mathbf{1}}$, González-Peñas $\mathbf{E}^{\mathbf{2}}$, López de Cerain $\mathbf{A}^{\mathbf{1}}$ \\ ${ }^{1}$ Departament of Pharmacology and Toxicology. ${ }^{2}$ Departament of Organic and \\ Pharmaceutical Chemistry, Faculty of Pharmacy. University of Navarra \\ Irunlarrea 1, 31080 Pamplona. \\ E-mail: avettora@unav.es. Phone:+34948425600
}

\begin{abstract}
Ochratoxin A (OTA) is a thermostable mycotoxin that contaminates a great variety of foodstuffs. It is nephrotoxic in all of the mammalian species tested, being the pig the most sensitive one; among rodents, rats are the most susceptible to OTA carcinogenicity. Kinetics, by studying the absorption, distribution, metabolism and excretion of xenobiotics, is an important tool for the extrapolation of animal toxicity data. The most important kinetic studies performed with OTA in rats have been reviewed, together with the different methods used for OTA quantification in biological matrices. Thirteen studies in Wistar, Sprague-Dawley or F344 rats, using radiolabeled OTA or TLC, HPLC-FLD or HPLC-MS have been summarized. Very often methods validated for food have been directly applied to tissues. Strain, sex and age differences have been detected but the interpretation is difficult due to the different experimental conditions, and the connection of the several factors that may account for these differences.
\end{abstract}

Keywords: ochratoxin A, kinetics, analytical techniques, rat

Short title: Ochratoxin A kinetics in rats 


\section{INTRODUCTION}

Ochratoxin A (OTA) is a mycotoxin produced by fungi of the genera Aspergillus and Penicillium that contaminates a great variety of foodstuffs, such as cereals, coffee, cocoa, beans, grapes and dried fruit. Due to the fact that OTA is not destroyed by common food preparation procedures, humans and farm animals are continuously exposed to OTA through the consumption of contaminated food and feed. Ochratoxin A is nephrotoxic in all of the mammalian species tested to date. Regarding OTA nephrotoxicity the pig is the most sensitive species; risk assessment is currently based on this animal (EFSA, 2006; WHO, 2008). However, with regard to OTA carcinogenicity, rodents are much more sensitive (NTP, 1989) (Bendele et al., 1985), being rats considered as more sensitive than mice, and rat males more sensitive than rat females (EFSA, 2006).

The main concern regarding ochratoxin A toxicity is its potential carcinogenicity to humans. The lack of human epidemiological data and the fact that the mode of action of OTA as a carcinogen is still unknown, make risk assessment difficult. Direct genotoxic (DNA binding of OTA moiety) and epigenetic mechanisms (non-genotoxic; thus, without any direct DNA binding of OTA moiety), have been proposed and evidence has been generated in support of each mechanism of action. This debate appears to be a key question for the selection of relevant studies and methods to be used for risk assessment (WHO, 2008). Understanding the mechanism underlying renal diseases in experimental animal will aid, not only in the elucidation of the mechanism of action, but also in the extrapolation to human situation and risk assessment.

An important tool that aids in the interpretation of toxicologic findings in animals and extrapolation of the resulting data to humans is kinetics, which is the science that studies the 
absorption, distribution, metabolism and elimination of a substance in the body. The relevance of toxicokinetics in toxicity evaluation and interpretation of animal toxicology data and its importance for a correct risk assessment has been reviewed by Dixit et al. (2003).

Thus, this article will briefly review the most important kinetic studies performed with OTA in rats; the most sensitive species regarding OTA carcinogenicity (EFSA, 2006) as well as the preferred species for toxicokinetic studies as it has been used extensively for toxicological studies of OTA and other xenobiotics (OECD, 2010). Moreover, due to the relevance of using sensitive, accurate and precise analytical methods in obtaining reliable kinetic data, the different extraction and quantification methods used for the measurement of OTA in biological matrices from rats in the different kinetic studies have also been reviewed.

\section{Analytical techniques for ochratoxin A toxicokinetic studies}

There are numerous methods for quantifying OTA, particularly in food matrices, that have been reported in the reference literature (EFSA, 2006; WHO, 2001). However, very few analytical techniques have been specifically validated for application in biological samples obtained from rats (Han et al., 2013, Vettorazzi et al., 2008; Zepnik et al., 2003). In many cases, the entire validation data has not been reported or the methods have been developed and validated in other animal species, such as a pig, or even in food commodities, and then applied to rat samples (Alvarez et al., 2004; Aoudia et al., 2008; Domijan et al., 2005; Kerkadi et al., 1998; Li et al., 1997; Mantle, 2008; Miljkovic et al., 2003). This could be acceptable for some objectives but, as reported in the S3A ICH Guideline for drugs (ICH S3A), the analytical technique method used in kinetic studies should always be validated in each type of sample and in each animal species in order to investigate possible interferences by endogenous components. This would also be relevant to other xenobiotics, especially in the case of OTA, in which several species-related differences have been observed. The need 
for a specific validation for each matrix has been demonstrated for OTA in a European intercomparison study (Entwisle et al., 1997), where several laboratories reported that, when using a method adapted from cereals, recoveries from the freeze-dried pig kidney material were lower than those obtained from cereals.

The main chromatographic methods for determination of OTA in animal and human tissues and fluids have been extensively reviewed by Valenta (1998). In the present review, only the methods used for quantifying OTA in kinetic studies performed in rats to date will be presented. Table 1 shows the extraction procedure and the detection method used on each organ of each kinetic study performed. Some important aspects regarding the analytical technique that may affect the interpretation of the kinetic results are mentioned below.

As can be observed in table 1, the former kinetic studies (Galtier et al., 1979; Kane et al., 1986; Suzuki et al., 1977) have quantified OTA in different biological matrixes either by thin layer chromatography (TLC) or by administering radiolabelled OTA to the experimental animals. The main advantage of the aforementioned studies is that OTA has been determined in a high number of organs. However, the analytical techniques used in these studies present some disadvantages when compared to newer analytical techniques, such as HPLC-FLD or HPLC-MS, used in the more recent studies. In the case of using radiolabelled OTA, not all of the radioactivity detected necessarily represents OTA because a certain proportion must also originate from degradation products, such as phenylalanine or OT $\alpha$. Moreover, the use of this method makes it more difficult to determine the absolute amounts per tissue weight (Schwerdt et al., 1996), as can be observed in table 2. Generally, TLC methods have higher detection limits and higher relative standard deviations in quantitative analysis than HPLC (Valenta, 1998). 
The most recent kinetic studies have used HPLC-FLD or HPLC-MS/ for OTA quantification in different rat biological samples. HPLC-FLD is the most widely used procedure for OTA determination in biological matrices due to the low detection limits and the high sensitivity that can be achieved, as OTA is a substance with high natural fluorescence (Valenta, 1998). Liquid chromatography detection methods and mass spectrometry are becoming more and more important for OTA in biological samples, especially for OTA metabolite detection (Muñoz et al., 2009; Schaut et al., 2008). In the main rat kinetic studies performed to date with HPLC-FLD or HPLC-MS, complete data from the validation for each matrix is not given or the extraction and/or detection methods were developed for other species (Li et al., 1997; Mantle et al., 2008; Zepnik et al., 2003). In some kinetic studies in which a specifically validated method was used, only plasma, kidney and liver levels were measured (Vettorazzi et al., 2009, 2010, 2011). Recently a new validated HPLC-MS method has been developed for uncovering in vivo kinetics of OTA in rats in plasma, heart, liver, spleen, lung, kidney and brain (Han et al., 2013).

Several aspects must be taken into account when determining OTA in biological samples (Valenta, 1998). Extraction from the biological sample is one important part of OTA quantification. Due to its protein binding properties, it is convenient to extract OTA in acidic conditions. Several authors have recommended that the $\mathrm{pH}$ of the sample solution should be adjusted to less than 2 in order to ensure a complete extraction of OTA from matrices rich in proteins (Pfohl-Leszkowicz and Manderville, 2007; Valenta, 1998). However, it should be mentioned that these acidic conditions were not reached in other studies using HPLC-MS and where high OTA recoveries were also obtained (Zepnik et al., 2003; Han et al., 2013). Han et al. (2013) compared three extraction solvents (methanol, acetonitrile and acetone) in absence of acidic conditions, and concluded that all three performed well (recoveries higher than 70 
$\%)$ as long as the ratio extraction solvent:sample was higher than 1:1 for methanol and acetonitrile; and 3:1 for the acetone..

However, the use of acidic extraction is under debate, especially regarding OTA metabolites extraction. In the study performed by Zepnik et al., 2003, no OTA metabolites (OTQ, OTHQ, open OTA lactone, hidroxilated OTA or glutathione metabolites) were detected in urine, liver, kidney and blood. Only OT $\alpha$ was detected in urine (no acidic extraction) and feces (acidic extraction); as well as traces of hexose and pentose OTA conjugates in urine. Some authors (Pfohl-Leszkowicz and Manderville, 2007) by using the same detection method, HPLCspectrofluorimetry, compared the method of extraction used by Zepnik et al. (2003) (only based on ethanol precipitation of proteins and the classical one that uses chloroform after acidification, finding that OTA metabolites were most probably lost during the ethanol deproteinization step. With the acidified chloroform extraction, they were able to detect compounds corresponding to the elution time of the 4-hydroxy derivatives $(4(R)$ and $4(S)$ OH-OTA), ochratoxin B (OTB), ochratoxin $\alpha(\mathrm{OT} \alpha)$, ochratoxin $\beta(\mathrm{OT} \beta)$ and the lactone opened ring of OTA (OP-OTA). However, it has been reported by Li et al. (2000) that low pH does not allow the detection of some lactone-opened forms of ochratoxins. In a more recent study (Han et al., 2013), by using LC-TOF/MS, metabolites such as phenylalanine, OT $\beta$ or OTB methyl ester, were detected in different tissues after methanol extraction. Thus, it seems there is lack of consensus regarding OTA metabolite extraction/detection. Moreover, even if practically all of the studies have synthesized the metabolites following similar protocols (mainly Dai et al., 2002; Xiao et al., 1996a; and Xiao et al., 1996b), there is a need for OTA metabolite standards. Finally, it would also be interesting to validate simple methods, either for OTA or its metabolites, for obtaining comparable data among different matrices. This was performed for OTA, and the complete validation results were published for HPLC-FLD by Vettorazzi et al. (2008) for plasma, kidney and liver and for many other tissues in HPLC-MS 
by Han et al. (2013). To validate the method for each individual matrix is especially relevant when HPLC-MS methods are used, as matrix effects can significantly affect the accuracy of the results. For this purpose, the use of internal standards is highly recommended. This aspect was also taken into account in the method validated by Han et al., (2013).

Another important aspect when dealing with biological matrices is the procedure used for spiking the samples. At present, there is a lack of certified reference materials for the determination of OTA. There is only certified material for blank wheat (European Union Joint Research Centre, Institute for Reference Materials and Measurements, Geel, Belgium). In recovery experiments, blanks samples must be spiked with a standard solution of the toxin prior to extraction. For biological samples, Valenta (1998) recommended spiking the sample with a small volume of standard solution in a solvent which is miscible with water, and mixing the sample to ensure good distribution of OTA in the sample. However, the author highlighted the fact that the results of recovery experiments cannot be applied to naturally contaminated samples without restrictions. Frequently, OTA recovery from samples has not been calculated and/or results have not been corrected by it. This, together with the fact that the spiking procedure is only described in a few methods, makes the comparison among different studies quite difficult.

Finally, even if in the most recent kinetic studies, the volume of sample needed for OTA extraction is low (table 1), it would be interesting to validate techniques in which smaller volumes, especially for plasma, are needed. This would allow obtainment of a better description of the kinetic curve with a reduction in the number of animals. The more data points that are obtained from one single animal, the more accurate the kinetic modeling is. Furthermore, it would enable the estimation of both inter-animal and residual variability when a population modeling approach is applied (Vettorazzi et al., 2009). 
A very common practice in kinetic analysis of OTA is the no inclusion of control animals. In all of the kinetic analyses performed to date with OTA, only Kane et al. (1986), Suzuki et al. (1977) Vettorazzi et al. (2009, 2011) and Han et al. (2013), included control animals. As stated by the EMEA/CPMP/SWP/1094/04 Guideline, control sampling and analytical procedures should be integrated into the toxicokinetic evaluations in order to check for possible contamination between samples during the in vivo experiment or during sample preparation. Significant contamination of controls with the test substance may ultimately invalidate the study. However, it is important to point out that, in the case of OTA, detectable levels can be observed in plasma from animals that were never in contact with OTA-treated animals (Vettorazzi et al., 2008). These low levels of OTA were also found in blank samples (Aoudia et al., 2008; Mantle, 2008) and have been attributed to the OTA contamination normally present in the standard diet of the animals.

Thus, it would be very interesting to give at least the same relevance to the analytical techniques used for toxicokinetic and toxicology studies as to the ones used in food matrices (EFSA, 2006; WHO, 2001; WHO, 2008), in order to assure reliable and comparable data among studies and consequently, a more accurate interpretation of the results. 


\section{OCHRATOXIN A KINETIC STUDIES IN RAT}

\section{Absorption}

It has been reported that OTA is rapidly absorbed after oral ingestion in several animal species (EFSA, 2006; WHO, 2001). In rats, Galtier et al. (1979) described a rapid absorption phase $(18 \mathrm{~min})$ for oral administration. OTA is mainly considered to be passively absorbed through the GI tract (reviews of Pfohl-Leszkowicz and Manderville, 2007; Ringot et al., 2006), through the stomach (Galtier, 1977) and particularly, through the proximal jejunum (Kumagai and Aibara, 1982). The passive absorption is highly favored by the high binding affinity of OTA to plasma proteins (Ringot et al., 2006). However, absorption from the jejunum can also take place against a concentration gradient and depends on the $\mathrm{pH}$ at the mucosal surface of the jejunum (Kumagai, 1988; WHO, 2001). Several substances, such as cholestyramine (Madhyastha et al., 1992) or micronized wheat fibers (Aoudia et al., 2008), have been shown to reduce the bioavailability of OTA when administered in the feed.

Only two studies have been specifically designed to allow comparison between oral and intravenous administrations, and thus allowing bioavailability calculation for OTA after oral administration. Galtier et al. (1979) calculated a bioavailability of $67.3 \%$ while Hagelberg et al. (1989) obtained a $44 \%$. However, it is important to note that the dose administered by Galtier et al. (1979) was 50 times higher than the dose administered by Hagelberg et al. (1989). It is well known that OTA can alter barrier and absorption functions of the intestinal epithelium, and even potentiate its own absorption through paracellular pathways (Maresca et al., 2001; Subramanian et al., 1991). Moreover, Suzuki 
et al. (1977) observed catarrhal enteritis produced by OTA or OT $\alpha$ through small intestine after a single oral dose of $15 \mathrm{mg} / \mathrm{kg} \mathrm{b.w}$. of OTA. They stated that the patterns of absorption, tissue distribution and excretion were affected by the enteritis. BreitholtzEmanuelsson et al. (1995), after a single intratracheal administration of OTA, calculated (by comparing their results with the ones obtained after intravenous administration by Hagelberg et al., 1989) a bioavailability of $98 \%$. The authors stated that the toxicokinetics of the toxin when given intratracheally, orally or intravenously were comparable.

In general, for a same dose, F344 females tend to reach higher plasmatic concentration than males (Vettorazzi et al., 2009; Zepnik et al., 2003) (table 3). The concentration of OTA in plasma also tends to be influenced by the age of the rats; younger rats (10 weeks old) reached higher OTA concentrations than older rats (15 weeks old) (Vettorazzi et al., 2009). The lower OTA plasma levels obtained in males coincide with other studies in which the higher maximum concentrations were found in Fischer X Sprague Dawley females after 5 months of OTA administration via diet (Mantle, 2008). Moreover, within $96 \mathrm{~h}$ after oral administration, Zepnik et al. (2003) detected a higher OTA fecal excretion in males than in females. This may suggest a possible less efficient gastrointestinal absorption in males; however, more experiments, in which biliar excretion can be compared between males and females, are needed.

Regardless of the administered doses, the maximum OTA concentrations observed after oral administration were between 1 and 7 hours in all of the studies in which OTA was administered dissolved in an aqueous vehicle (tables 2 and 3). Upon comparing two toxicokinetic studies in which a same dose of OTA was administrated in corn oil (Zepnik et al., 2003) or in $\mathrm{NaHCO}_{3}$ (Vettorazzi et al., 2009), similar OTA concentrations in plasma 
were reached at $48 \mathrm{~h}$ in males and at $24 \mathrm{~h}$ in females. However, in Zepnik et al. (2003) these levels were the maximal concentrations reached in the curve, whereas in Vettorazzi et al. (2009) the maximal OTA concentrations were attained $2 \mathrm{~h}$ (for males and young females) and $6 \mathrm{~h}$ (for mature females) after the oral administration (table 3). This difference could be due to the possibility that the corn oil might delay absorption of OTA or to the lack of data in earlier timepoints after administration where higher OTA levels could have been detected.

Another factor that may influence OTA absorption is the presence of food in the stomach of the animals before the administration. In Vettorazzi et al. (2010), after performing a $24 \mathrm{~h}$ kinetic study in fasted rats and comparing the results with fed rats, it was found that fasting tends to increase the maximum plasma concentrations and the rate of absorption in both males and females. This difference was only statistically significant in male rats that consumed more food than the rest of the groups (females and young males). However, food ingestion was proportional to body weight in all experimental groups. The reason why the OTA bioavailability is more affected in the presence of food only in males is unknown. Some possible consequences of this higher amount of food in the stomach of males, such as delayed gastric emptying or the trapping effect of the food contents towards the mycotoxin have been commented in Vettorazzi et al. $(2010,2011)$. The fact that food in the stomach may lead to less OTA absorption coincides with the fact that OTA appears to be less toxic when acquired as a dietary component (Mantle et al., 2005; Miljkovic et al., 2003).

Finally, another factor to be taken into consideration is the possibility of a carrier-mediated transport in gastro-intestinal tract. Even if it has been demonstrated in Caco-2-cells that organic anion transporters (OAT) are not involved in OTA transport across the cells 
(Berger et al., 2003), the fact that absorption from the jejunum can also take place against concentration gradient (Kumagai, 1988) suggests the presence of transporters with the capacity to transport OTA in the jejunum (Dietrich et al., 2005). This, together with the fact that OTA may be a substrate for the ATP-Binding Cassette efflux-proteins MRP2 (multidrug resistance-associated protein) and BCRP (breast cancer resistance protein) in Caco-2-cells (Schrickx et al., 2006) (see "elimination" section), may influence the bioavailability of OTA.

\section{Distribution}

Once OTA is absorbed, it binds to serum albumin (Galtier et al., 1981) and other macromolecules (Stojković et al., 1984). The unbound fraction is as low as $0.02 \%$ in rats and in humans, indicating an extent of plasma protein binding of $99.98 \%$ (Hagelberg et al., 1989). This binding facilitates its passive absorption in the non-ionized form and partly explains its long half-life in the body (Dietrich et al., 2005; Ringot et al., 2006). Hagelberg et al. (1989) also studied the relationship between plasma binding properties of OTA and its toxicokinetics in several animal species; considerable differences were found between species. Indeed, care should be taken when interpreting OTA kinetic data from different rat strains, as marked strain-differences in binding affinity of albumin to other xenobiotics have already been described (Ito et al., 2007).

Stojkovic et al. (1984) found that in human and porcine serum, OTA binds more specifically to small proteins (molecular mass around $20 \mathrm{KDa}$ ) than to albumin. Due to the

fact that such small proteins can pass through the glomerular membrane, the authors concluded that this binding could be relevant to the OTA predominant nephrotoxic effect 
on mammals. More recently, Mantle and Nagy (2008) suggested that the low molecular weight protein $\alpha 2 \mathrm{u}$-globulin (with a molecular mass also around $18 \mathrm{KDa}$ ) may be involved in some of the OTA differences found between male and female rats. The $\alpha 2 \mathrm{u}$-globulin is a male rat-specific protein that is synthesized in the liver of males under androgenic control and reaches very high levels in plasma and urine of adult male rats. It is known that many chemicals that only cause renal tumors in male rats, such as d-limonene or 2,2,4trimethylpentane, induce accumulation of the $\alpha 2 \mathrm{u}$-globulin in the kidney (for a review see Swenberg, 1993). This accumulation initiates a sequence of events that appears to lead to nephropathy and renal tumor formation (Rodgers and Baetcke, 1993). Even though it has been demonstrated that OTA kidney lesions are different from the $\alpha 2 \mathrm{u}$-nephropathy in all characteristic points (Rasonyi et al., 1999) and that OTA also causes renal tumors in male mice (Bendele et al., 1985), Mantle and Nagy (2008) suggested that the $\alpha 2 \mathrm{u}$-globulin could act as a specific OTA carrier that increases proximal tubule exposure to the mycotoxin in male rats. However, this has not been demonstrated experimentally.

The fraction of OTA bound to serum albumin and other macromolecules constitutes a mobile reserve of mycotoxin that can be made available for release to the tissues for a long time and partially explains the long half-lives obtained in different OTA kinetic studies (tables 2 and 3). Some authors considered that half-life after oral ingestion is shorter than after intravenous injection (Pfohl-Leszkowicz and Manderville, 2007; Ringot et al., 2006). However, this has been observed in rats in only one study (Hagelberg et al., 1989). In another study performed with both routes of administration (Galtier et al., 1979), this difference in half-lives was not observed. Moreover, the aforementioned authors explained the shorter elimination half-life found in orally dosed rats, as a reflection of the first pass 
effect with primary biliar and subsequent fecal excretion. However, a high first pass effect may explain the low bioavailability of OTA after oral ingestion rather than differences in half-life between i.v. and p.o administrations. The half-life is a kinetic parameter which directly depends on the volume of distribution and clearance, parameters that are not generally affected by any loss of the substance (such as in the first pass effect) before entering to the systemic circulation. Dietrich et al. (2005) stated that the observed differences in elimination half-lives between oral and i.v application routes most likely stem primarily from analytical limitations rather than from real biological effects. For example, the half-lives obtained by Galtier et al. (1979) were shorter than the ones reported in the rest of the studies, probably due to the different technique that was used to quantify OTA in the biological matrices or to the lack of timepoints (samples were only collected during the first $48 \mathrm{~h}$ ). However, it should be noted that the majority of the OTA kinetic studies performed with Sprague-Dawley rats obtained shorter half-lives than the studies carried out with other rat strains (table 3). The impact of the different rat strains in OTA kinetics remains unclear.

Another factor that should be taken into account is the body weight of the animals used in the different studies. In a study in which kinetic analysis were performed in F344 rats of different ages and sexes (Vettorazzi et al., 2009), a statistically significant impact of body weight on volume of distribution, which increased linearly with body weight, was found. Due to the fact that the half-life is governed by both plasma clearance and volume of distribution, it was concluded that the differences observed in the half-lives between the different groups of rats were due to differences in body weight; no differences in the clearance between groups were observed. This study highlighted the importance of taking 
into account the age, weight and sex, when studying OTA toxicokinetics in F344 rats. This coincides with one of the studies performed by Mantle (2008) in which Dark Agouti and F344 males of the same age were administered the same OTA dose. The body weight and OTA half-life (2.16 days) of Dark Agouti rats were less than those of F344 (8.64 days). However, again, other strain differences, and not only body weight, may be involved in such a big difference between half-lives.

With regard to tissue distribution, previous data from in vivo experiments had indicated that OTA accumulates in the kidney, its main target organ (EFSA, 2006). The tissue distribution of OTA in rats follows a different order, depending on the technique used to quantify OTA and the experimental design of the kinetic study (Galtier et al., 1979; Han et al., 2013; Kane et al., 1986; Li et al., 1997; Suzuki et al., 1977). Studies in rats by Chang and Chu (1977) and Suzuki et al. (1977) also demonstrated accumulation of the toxin in the gastrointestinal tissues. OTA has also been found in brain (Belmadani et al., 1998; Han et al., 2013) and is able to cross the placenta (Hallen et al., 1998). In general, kidney, heart and liver are the organs in which higher amounts of OTA are found. OTA concentrations in liver and kidney tend to be similar between both organs (in some cases higher in liver than kidney) after both single and repeated exposure (Aoudia et al., 2008; Arbillaga et al., 2008; Breitholtz Emanuelsson et al. 1991; Domijan et al., 2005; Galtier et al., 1979; Han et al., 2013; Kane et al., 1986, Suzuki et al., 1977, Tozlovanu et al., 2012, Vettorazzi et al., 2011). However, Zepnik et al. (2003) found much higher OTA concentrations in kidney than in liver (40-, 100- and 270-fold higher in kidney than in liver in male rats at $24 \mathrm{~h}, 48 \mathrm{~h}$ and $72 \mathrm{~h}$ ) after the single oral dose administration. The main difference between Zepnik et al. (2003) and the aforementioned studies is that an acidification step was not used in the 
extraction method (see "Analytical techniques for ochratoxin A toxicokinetic studies" section). However, when the same analytical method was applied in samples from repeated dose studies (Mally et al., 2005; Rached et al., 2007), similar levels of OTA were found between both organs. The authors explained the similar concentration between both organs at higher doses as being due to an effect of redistribution and deposition of OTA in all lipid-rich organs. Similar results were obtained in the study performed by Han et al., 2013 after extraction with methanol: a higher concentration of OTA in kidney than in liver after a single oral dose was found, but almost equal between both organs after a repeated oral dose. However, in this case, the authors attributed this finding to the higher amount of vascular tissue in liver than in kidney and to the fact that the concentrations in the different tissues were positively related to plasma concentrations after the single oral dose, in agreement with other studies (Vettorazzi et al., 2011)

With regard to sex differences, in Vettorazzi et al. (2011), it was observed that the differences found in kidney and liver concentrations between males and females of different ages and in different fast/fed conditions before the OTA oral administration correlate with the plasma profiles of each different group. This demonstrates the parallel concentrations of OTA in plasma and tissues and therefore, reveals that the sex differences observed in organs are both a reflection of the differences in bioavailability observed and the significant increment that occurs in $\mathrm{V}$ and $\mathrm{V}_{\mathrm{L}, \mathrm{K}}$ when body weight increases. However, Zepnik et al. (2003) observed a much higher OTA accumulation in kidney of male rats than in females. Once again, this contradiction between results highlights the need of taking into account the analytical technique used for OTA quantification: non-acidic conditions may produce inefficient protein separation, leading to an underestimation of OTA concentration 
and yet, the deproteinisation step with ethanol/acidic conditions may interfere with some metabolites detection.

As commented above, the kidney is the main target organ of OTA. However, OTA has been detected in many other organs (mainly liver, lung and heart) at similar or even higher levels after OTA administration. Thus, as stated by Han et al. (2013), OTA toxicity is not only driven by tissue distribution and kinetics, but also by organ-specific toxicodynamics. It had been suggested that the marked differences in the relative sensitivity of individual species and sexes towards OTA might be due to variations in the transport mechanisms and cellular uptake in renal cells (EFSA, 2006). Furthermore, species- and sex-specific expression of OTA-specific transporters could modulate organ and cellular OTA concentrations and kinetics and therefore, have a direct influence on toxicodynamics (Dietrich et al., 2005). It is known that OTA is a substrate for the family of organic anion transporter proteins (OATs) and organic anion transporting polypeptide (OATP) (Anzai et al., 2005; Babu et al., 2002; Takeuchi et al., 2001; Tsuda et al., 1999) and that it can even regulate the expression of these transporters in rat kidney cortex (Zlender et al., 2009) or impair the activity of the organic anion transporters (Sauvant et al., 1998). The localization, molecular and functional characteristics, and substrate and inhibitor specificity of the main organic anion transporters have been extensively reviewed (Russel et al., 2002; Wang and Sweet, 2013). These specific transporters may also be involved in the accumulation of OTA in organs other than kidney (Anzai et al., 2010, Eckhardt et al., 1999; Kontaxi et al., 1996, O'Brien and Dietrich 2005). It is also important to highlight that OTA kidney uptake and handling of OTA by OATs is dependent on the OTA bound to plasma protein (Bow et al., 2006). 


\section{Metabolism}

The information regarding the metabolism of OTA remains unclear and controversial. The complete biotransformation pathways of OTA are still unknown. Some of the metabolites have been characterized in vitro and/or in vivo, while other metabolites remain to be characterized (Ringot et al., 2006). As commented above, there is a need to develop good and validated analytical techniques for quantifying OTA metabolites in the different tissues and biological fluids. The metabolism of OTA has been extensively reviewed by Ringot et al. (2006), Pfohl-Leszkowicz and Manderville (2007) and Wu et al. (2011). An important aspect to take into account when studying the OTA metabolites in rats is the controversial interpretation of the results observed among studies (Mally and Dekant, 2009; PfohlLeszkowicz and Manderville, 2007). While some authors have observed the formation of several different OTA metabolites in the rat (Castegnaro et al., 1989; Li et al., 2000; Xiao et al., 1996a; Xiao et al., 1996b) and considered them to be reactive metabolites (Dai et al., 2002; Obrecht-Pflumio et al., 1996; Pfohl-Leszkowicz et al., 1998; Pfohl-Leszkowicz and Castegnaro, 2005), others have considered that the biotransformation of OTA is very low (Gautier et al., 2001; Gross-Steinmeyer et al., 2002; Zepnik et al., 2001; Zepnik et al., 2003) and unlikely to form reactive metabolites. The reason for these differences still remains an open question. The different metabolites identified in the different studies may stem from the different analytical techniques used: radiolabeling + TLC; HPLC-FLD or HPLC-MS and if a complete validation of the method has been carried out (see section * Analytical techniques for ochratoxin A toxicokinetic studies“). Indeed, HPLC-FLD does not allow structural identification of the metabolites compared with methods using HPLCMS. However, some authors using HPLC have used a second confirmation step 
(conversion of the metabolites into other forms) (Li et al., 2000) while only one study (Han et al., 2013) using HPLC-MS have applied internal standards to take into account the matrix effect (which directly affects the accuracy of the HPLC-MS results). On the other hand, as commented previously (in section Analytical techniques for ochratoxin A toxicokinetic studies), the use of acidic or non-acidic extraction might directly affect the recoveries of certain metabolites. Finally, another factor that should also be taken into account is the origin of the samples used for metabolites identification: organs and tissues collected and in vivo experimental design. Some studies used samples from single oral administration studies but using different vehicles and rat strains: $0.5 \mathrm{mg} / \mathrm{kg}$ bw in corn oil in F344 rats (Zepnik et al., 2003) and $0.2 \mathrm{mg} / \mathrm{kg}$ b.w in water/ethanol solution in Sprague Dawley rats (Han et al., 2013). Others used a single intravenous administration $0.33 \mathrm{mg} / \mathrm{kg}$ b.w in saline/ethanol solution in Sprague Dawley rats (Li et al., 2000) or repeated oral doses by gavage $2 \mathrm{mg} / \mathrm{kg}$ b.w in corn oil for 2 weeks in F344 rats (5 days/week) (Mally et al, 2004) or in OTA diet for 28 days in Dark Agouti rats (Tozlavanu et al. (2012). These different experimental designs should also be taken into account when interpreting the different metabolites obtained, as the vehicle, the dosage regimen and the rat strain used may affect the type and the amount of metabolite detectable by each analytical technique. Thus, more studies that take into account the aforementioned variables are needed. .Figure 1 shows the most important metabolites detected in OTA-treated rats throughout different in vivo studies.

The major metabolite pathway of OTA consists in its detoxification by hydrolysis into OT $\alpha$ (figure 1), a much less toxic compound, as well as easily eliminated from the body. OT $\alpha$ is the dihydroisocoumarin derivative produced by the cleavage of the peptide bond of OTA. 
In rats, the hydrolysis to OT $\alpha$ is a function of the bacterial microflora of the caecum. The enzymes responsible for the hydrolysis to OT $\alpha$ are carboxypeptidase A, and possibly trypsin, $\alpha$-chymotrypsin and cathepsin C (Galtier, 1991). OT $\alpha$ is mainly excreted in feces, however the metabolite was also found in urine (table 2 and Storen et al., 1982). Its presence in the urine can be explained by reabsorption from the intestine (WHO, 2001). Suzuki et al. (1977) showed that the duodenum, ileum and pancreas have greater capacity for hydrolyzing OTA, whereas the activity in the kidney and liver was low. Galtier et al. (1979) also confirmed this result. OT $\alpha$ was also detected in plasma, urine and feces in a more recent kinetic study performed by Zepnik et al. (2003). However, the metabolite OT $\alpha$ was not present in solid organs in some studies (Zepnik et al., 2003; Han et al., 2013), but detected in liver and kidney in others (Tozlavanu et al., 2012).

A small percentage of absorbed OTA is also detoxified, mainly in the liver through the action of CYP450, into the hydroxylated derivatives 4(R)- and 4(S)-OH-OTA through phase I detoxification reactions (figure 1$)$. The $4(R)$-OH-OTA epimer is mainly formed by human and rat liver microsomes whereas 4(S)-OH-OTA epimer is mostly formed by pig liver microsomes (Ringot et al., 2006). Several studies in different experimental systems pointed out that different CYP450 isoforms may be involved in the formation of each epimer and other metabolites (for a review check Pfohl-Leszkowicz and Manderville, 2007, WHO, 2001). OTA hydroxylated derivatives have also been produced in rat kidney (WHO, 2001).

Other OTA metabolites have also been found in the rat. Ochratoxin B (figure 1), the dechloro derivative of OTA that is much less toxic than OTA (Mally et al., 2005) has been detected in urine of OTA-treated rats (Mally et al., 2004). Li et al. (2000) demonstrated the 
presence of a lactone-opened OTA (OP-OTA) (figure 1) in urine and bile of female Sprague-Dawley rats administered OTA intravenously. It has been demonstrated that the OP-OTA is highly toxic when administered i.v. in rats (Xiao et al., 1996a). The OTAderived quinones (OTQ/OTHQ) (figure 1) and their glutathione conjugate (OTHQ-GS) have been hypothesized as possible reactive metabolites (Dai et al., 2002; Gillman et al., 1999) and related with the genotoxic effects of OTA (Pfohl-Leszkowicz and Manderville, 2012). The presence of O-labile ester conjugates of OTA (figure 1) was also found in the urine of F344 rats after a single oral dose of OTA (Zepnik et al., 2003). The authors suggested that these two compounds could be formed in an enzymatic process in which alpha-glucosidases of the liver may be involved. However, in this study, except for OT $\alpha$, other OTA metabolites such as OTQ, OTHQ, OP-OTA and glutathione conjugates and glucuronides of OTA were not present in urine, liver, kidney, or blood samples. However, the OTHQ metabolite was detected in trace amounts in urine of male F344 rats treated with a repeated (2 weeks) oral dose of $2 \mathrm{mg} / \mathrm{kg}$ b.w of OTA (Mally et al., 2004). It should be highlighted that the mass spectra of the hydroquinone derivative (detected by Mally et al., 2004) might be very similar to that of hydroxylated ochratoxin B (related to the OTB detected by Han et al., 2013). Thus more studies are needed to understand the possible commonalities among the few studies evaluating in vivo metabolism of OTA. In a more recent study (Tozlavanu et al., 2012) in which Dark Agouti rats were fed various concentrations of OTA in wheat for a period of 28 days, several glutathione conjugates, such as OTHQ-GSH and OTB-GSH were detected by HPLC-fluorescence detection. Indeed, the glutathione conjugates were proposed by the authors as good biomarkers of exposure. In another recent study (Han et al., 2013) the only metabolites detected by LC- 
TOF-MS in all the tissues analyzed after a single oral dose administration of OTA, were phenylalanine in heart and kidney; OT $\beta$ in kidney and OTB methyl ester in spleen. The fact that some metabolites can be formed during sample workup and not in vivo, should also be taken in account. For example, it has been demonstrated that overnight exposure to $2 \mathrm{M} \mathrm{HCl}$ can change OTA into OT $\alpha$ or that the lactone ring of OTA can be hydrolyzed after adition of $\mathrm{NaOH}$ in DMSO for $2 \mathrm{~h}$ at $25^{\circ} \mathrm{C}$ (Valenta et al., 1998). In the case of methyl- or ethylesters of OTA it has been demonstrated that OTA standards in methanol were stable for at least $5 \mathrm{~h}$ at room temperature, and for at least three weeks at $4{ }^{\circ} \mathrm{C}$ (Jiménez et al., 1998), and that standards or processed samples extracted with a solution 1:5:8 (20\% trichloroacetic acid: saline solution or sample: absolute ethanol) were stable for $7 \mathrm{~h}$ in the autosampler carousel (plasma samples) or for at least 24h (standards and kidney or liver extracts) (Vettorazzi et al., 2008).

Thus, as commented previously, the different types and yields of metabolites found in the different in vivo studies may be mainly due to the different analytical techniques and approached used for the metabolite detection. However, the involvement of the different metabolic capacities of the different rat strains used, as well as the different in vivo experimental designs (single vs repeated doses; routes of administration) may be affecting the results as well.

For example, in a two-year gavage study performed with Dark Agouti (DA) (males: extensive debrisoquine (DB) metabolizers; females: poor DB metabolizers) and Lewis rats (both sexes considered to be extensive DB metabolizers), a different susceptibility to OTAinduced tumorogenesis was found (Castegnaro et al. 1998). In another study (PfohlLeszkowicz et al. (1998) demonstrated that the strain- and sex-specific genotoxic response 
of OTA found in Castegnaro et al. (1998) was partially controlled by CYP-mediated metabolic reactions that convert OTA into a DNA-reactive intermediate. They found that in male DA rats, the OTA-toxifying enzymes (CYP450 2C11, 1A2, and 3A) were highly expressed in the liver while little of the detoxifying isoforms (CYP450 1A1 and 2A) was detected. They also suggested that the induction of CYP2C11, a debrisoquine metabolizing phenotype in rat, may also be relevant in the higher susceptibility to OTA carcinogenesis of DA male rats. They concluded that the strain- and sex-specific genotoxic response of OTA is controlled, in part, by CYP-mediated metabolic reactions that convert OTA into DNAreactive intermediates in kidney and liver.

However, it is also important to point out that basal CYP expression of untreated rats also showed great strain and sex differences. These sex and strain-related differences could be highly problematic, not only in kinetic studies of OTA, but also in toxicological tests. There is a need to provide insights into important aspects of the genetic background of animals to the researchers who use these experimental models (Sakai and Ishizuka, 2009).

\section{Excretion}

Fecal, biliar and renal excretion play an important role in plasma clearance of OTA (Ringot et al., 2006; WHO, 2001). Some authors considered that the biliar route is the predominant OTA excretion route in the rat while in humans and non-human primates the primary route of excretion is via the kidney (Dietrich et al., 2005). This statement is supported by the results obtained in F344 rats of both sexes (Vettorazzi et al., 2011) in which one of the models that best fitted to data was the one which assumed that OTA elimination occurs mainly from the liver compartment. In mammals, OTA can also be excreted via milk, and 
this has also been studied in the rat (Breitholtz-Emanuelsson et al., 1993; Hallen et al., 1998). The contribution of each route of excretion depends on factors such as the route of administration, the dose, the degree of serum macromolecular binding and differences in degree of enterohepatic circulation (Kuiper-Goodman and Scott, 1989). Storen et al. (1982) found that the major excretory products in urine and feces of rats were OT $\alpha$, OTA and 4(R)-OTA. Regardless of the route of administration (i.p. or p.o., $6 \mathrm{mg} / \mathrm{kg} \mathrm{b.w.} \mathrm{of} \mathrm{OTA}$ dissolved in $\left.\mathrm{NaHCO}_{3}\right), 6 \%$ of the dose was excreted as OTA, 1 to $1.5 \%$ as 4(R)-OTA, and 25 to $27 \%$ as OT $\alpha$ in the urine. However, only traces of OTA and OT $\alpha$ were identified in feces after i.p. administration, whereas after oral administration, $12 \%$ OTA and $9 \%$ OT $\alpha$ were found. After an oral administration, Suzuki et al. (1977) detected up to $33 \%$ of radiolabelled OTA in the bile of rats up to $6 \mathrm{~h}$ after dosing. OT $\alpha$ was not detected in the bile. Galtier et al. (1979) obtained higher OTA elimination via feces than via urine after an i.v administration of $2.7 \mathrm{mg} / \mathrm{kg}$ b.w. of ${ }^{14} \mathrm{C}-\mathrm{OTA}$ (table 2). After a single iv administration of OTA, Li et al. (1997) (table 3) estimated a biliar clearance which was lower than renal clearance. However, they reported a high metabolic clearance, which may, partially involve the liver. After calculating the clearance of OTA by renal filtration, Hagelberg et al. (1989) also considered that most of the OTA is eliminated by another route. In a more recent study, in which F344 rats of both genders were orally administered $0.5 \mathrm{mg} / \mathrm{kg} \mathrm{b.w}$. of OTA dissolved in corn oil, Zepnik et al. (2003) found that the recovery of unchanged OTA within $96 \mathrm{~h}$ in urine was only $2.1 \%$ and $5.2 \%$ of the dose in males and females, respectively. The highest concentrations of OTA and OT $\alpha$ in urine of male and female rats were reached in the interval between $12 \mathrm{~h}$ and $24 \mathrm{~h}$ after administration. The excretion of OT $\alpha$ in urine of male rats was more than three times higher than in females, but excretion 
of OTA of female rats in this interval was up to three times higher than in male rat. In feces, the percentage of the dose recovered was $5.5 \%$ and $1.5 \%$ in males and females, respectively.

With regard to fecal excretion, both OTA and OT $\alpha$ have been detected in feces. However, it is difficult to distinguish whether or not the levels of OTA and OT $\alpha$ detected in feces are due to non-absorbed OTA, to the intestinal OTA secretion or to biliar excretion. Berger et al. (2003) suggested that the absorption of OTA across the intestinal mucosa would be limited thanks to its excretion through MRP2 at the apical pole of enterocytes. Ringot et al. (2006) suggested that the MRP2 may also be involved in hepatobiliary elimination of OTA conjugates. Chandra and Brouwer, (2004) stated that MRP2 is responsible for the biliary excretion of organic anions including glutathione, glucuronide, and sulfate conjugates of xenobiotics.

Enterohepatic circulation of OTA has been demonstrated in several studies with rats (Fuchs and Hult, 1992; Kane et al., 1986; Kumagai and Aibara, 1982). This means that OTA or OTA-metabolites are secreted into bile which is excreted into duodenum via the common bile duct. Subsequently, they are excreted into the feces or they are reabsorbed and become systematically available. It is known that some substances can be excreted into bile as conjugates, and then hydrolyzed in the gut back to the parent substance through the action of the intestinal microflora. In this case, the substance becomes available for reabsorption. This has been demonstrated for OTA in mice (Roth et al., 1988). One enzyme that could be involved in this process is the $\beta$-glucuronidase which is produced by intestinal bacteria. Studies have shown that intestinal glucuronidase in the rat is extremely high in comparison with other species. Fuchs and Hult (1992) stated that differences in effectiveness of 
enterohepatic circulation of OTA among animal species might be partially responsible for the differences in the retention of the toxin in plasma among the species.

With regard to the influence of plasmatic proteins in the elimination of OTA, it has been demonstrated that the half-life of OTA in albumin-deficient rats is much shorter than in normal rats (Kumagai, 1985), and that the concentration of OTA in bile and urine was 20to 70 -fold times higher in the albumin-deficient rats than in control animals. As stated by Dietrich et al. (2005), these observations demonstrate the importance of plasma/protein binding for the biological half-life of OTA. Thus, differences in OTA affinity binding to proteins between sexes or strains, may lead to differences in OTA elimination. An example is the shorter OTA half-life obtained in Dark Agouti (DA) rats in relation to F344 rats (Mantle, 2008) or in Sprague-Dawley rats in relation to the other strains (Table 3). However, Mantle et al., (2008) stated that both, DA (Castegnaro et al., 1998) and F344 rats (NTP, 1989) developed renal tumors after 2 years of OTA exposure and considered that the rate of elimination from plasma may not be an important factor per se in determining renal disease due to OTA (Mantle, 2008).

CONCLUSIONS AND RECOMMENDATIONS REGARDING THE ANALYTICAL METHODS AND IN VIVO EXPERIMENTAL DESIGNS USED FOR OCHRATOXIN A KINETIC STUDIES IN RATS

Based on the revision of 13 scientific publications in which OTA kinetics have been evaluated it can be concluded than:

- Number of studies evaluated. From a total of 13 studies, 5 studies were performed before 1995 using techniques considered nowadays as not meeting current modern scientific quality standards (at least for quantification) such as radiolabeled OTA, TLC or spectrofluorimetry detection. As from 1997, all the studies reviewed (8) used HPLC-FLD or HPLC-MS for OTA quantification. The latter allows unequivocal identification of OTA and its metabolites, while the former needs some 
confirmation methods. HPLC-FLD is the most used technique until date for OTA quantification.

- Validation of the method. From these 8 studies only 3 of them studies have used an analytical technique validated for the specific type of sample analyzed (biological matrix and animal species). Only 2 of these 3 studies have reported the entire validation data: one for HPLC-FLD and one for HPLC-MS. Validation should consider at least the following criteria: selectivity, linearity, precision (within- and between-day variability), limits of detection (LOD) and quantification (LOQ), recovery, stability of the samples (during storage and after extraction when kept in the autosampler carousel). In the specific cases of HPLC-MS matrix effect should also evaluated and taken into account in the quantification of the samples. For that purpose, the use of internal standards is highly recommended. Finally, for a correct quantification (and comparison among studies) it is very important to calculate the recovery from the extraction procedure and to indicate if results have been or not corrected by recovery values. As nowadays there is still absence of OTA certified reference materials for biological matrices the spiking procedure should also be clearly described.

- Metabolites identification and quantification. Until date some efforts have been performed to identify OTA metabolites in vivo. However, there is still a need of validated methods for OTA metabolites quantification. For that purpose:

- the influence of the extraction method for the different metabolites should be further studied.

- metabolite standards or biological certified reference materials for metabolites should be available.

- The vehicle used for OTA administration, the dosage regimen (dose, single versus repeated administration, oral versus iv administration) and the rat strain/sex should be carefully selected. These factors might directly affect the type and the amount of metabolite detectable by each analytical technique.

- Volume of samples. When validating new analytical methods is important to use low volume of biological samples. This will allow OTA/metabolites quantification in each sample individually, preventing the need to mix plasma or tissues from different animals and allowing a more accurate kinetic analysis of the data.

- Strain. The in vivo studies evaluated in the present review seems to indicate that Sprague-Dawley rats have shorter half-lives than other strains (F344 or wistars). 
This should better characterized in order to better understand OTA toxicity in the different rat strains.

- Sex. Sex differences at different dosage should be further explored. When sexes differences are studied the age of the animals should also be carefully taken into account. Body weight differences increases with age between male and female rats and it has been found that there is a statistically significant impact of body weight on OTA volume of distribution.

- Fast/Fed conditions. The fasted or fed condition of the animals should be clearly stated in all the kinetics studies. Food intake has a direct effect on OTA kinetics, especially for male rats. The effect of food might interfere as well with the yield and type of metabolites produced in vivo.

- Kinetic timepoints. Early timepoints should be selected in order to evaluate the entire absorption process.

- Controls. Control animals (non-treated) should always be included in kinetic experiments in order to confirm that no contamination of control samples had occurred during animal administration or analytical sample preparation.

- Toxicodynamics. It is clear that OTA toxicity is not only driven by tissue distribution, as the mycotoxin has been detected at similar levels in other non-target organs (liver, heart and lung) than in the main toxicity target organ (the kidney). More studies, focused on understanding the kidney-specific toxicodynamics should be performed. The role of kidney transporters and plasmatic proteins (small proteins) on OTA kinetics at kidney level should be also be further explored for understanding sex and strain differences.

\section{CONCLUSION}

Strain and sex-differences in OTA kinetics in rats have been described, but they are complex and not easy to summarize. The wide variety of analytical techniques applied for OTA measurements, the different experimental designs used, and the fact that raw data is 
generally not published, makes comparison among studies difficult. Moreover, the age, weight and fasting/fed conditions of the animals could act as confounding factors and thereby, they should be taken into account when gender differences are studied. To aid in this analysis, three tables with the most relevant data obtained from the different published kinetic studies until now, have being prepared for this review.

Only the first kinetic studies, which used radiolabelling and TLC to quantify OTA in tissues, have analyzed OTA in a large number of organs. In general, later studies have included fewer tissues and timepoints, and one or two compartment models have been applied in all of them. Quantifying OTA in more tissues would allow the use of more complex models which could consider three or four compartments. Moreover, there is a lack of studies using more complex physiologically-based pharmacokinetic models in order to take into account the presence of active transport and protein binding.

Regarding sex differences, some studies in Wistar or Sprague-Dawley rats have used only males or only females and the different conditions make impossible the comparison; in F344 rats, four studies carried out with males and females have detected sex differences, but the reason behind this observation is not clear at all.

In order to obtain accurate results, the analytical technique used to quantify a xenobiotic in a biological matrix should be specifically validated in that matrix. Unfortunately, very few OTA kinetic studies have used specifically validated methods for each tissue and very often methods validated for food matrices have been directly applied to biological ones. Moreover, due to the high controversy regarding the mechanism of action of OTA, there is also a need for developing good analytical techniques to quantify OTA metabolites in 
biological matrices. However, great caution should be taken in the choice of the species/strain due to the known interspecies and inter-individual genetic variations on xenobiotic metabolism.

In conclusion, there are data that indicate strain, sex and age differences in OTA kinetics in rat, but a clearer understanding is needed to elucidate if this factor may account for the different sex and species sensitivities to OTA. Well designed kinetic studies, using validated analytical techniques for proper matrices, are necessary to aid in the interpretation of the toxicity studies.

\section{ACKNOWLEDGMENTS}

We thank the project of Research of University of Navarra (PIUNA). 


\section{REFERENCES}

Alvarez, L., Gil, A.G., Ezpeleta, O., Garcia-Jalon, J.A., Lopez de Cerain A., 2004 Immunotoxic effects of Ochratoxin A in Wistar rats after oral administration. Food Chem. Toxicol. 42: 825-834.

Anzai, N., Jutabha, P., Endou, H., 2010. Molecular mechanism of ochratoxin a transport in the kidney. Toxins: 2:1381-1398.

Anzai, N., Jutabha, P., Enomoto, A., Yokoyama, H., Nonoguchi, H., Hirata, T., Shiraya, K., He, X., Cha, S.H., Takeda, M., Miyazaki, H., Sakata, T., Tomita, K., Igarashi, T., Kanai, Y., Endou, H., 2005. Functional characterization of rat organic anion transporter 5 (Slc22a19) at the apical membrane of renal proximal tubules. J. Pharmacol. Exp. Ther. 315: 534-544.

Aoudia, N., Tangni, E.K., Larondelle, Y., 2008. Distribution of ochratoxin A in plasma and tissues of rats fed a naturally contaminated diet amended with micronized wheat fibres: Effectiveness of mycotoxin sequestering activity. Food Chem. Toxicol. 46: 871-878.

Arbillaga, L., Vettorazzi, A., Gil, A., van Delft, J., García-Jalón, J.A., López de Cerain, A., 2008. Gene expression changes induced by ochratoxin A in renal and hepatic tissues of male F344 rat after oral repeated administration. Toxicol. Appl. Pharmacol. 230: 197-207.

Babu, E., Takeda, M., Narikawa, S., Kobayashi, Y., Enomoto, A., Tojo, A., Cha, S.H., Sekine, T., Sakthisekaran, D., Endou, H., 2002. Role of human organic anion transporter 4 in the transport of ochratoxin A. Biochim. Biophys. Acta 1590: 64-75.

Belmadani, A., Tramu, G., Betbeder, A.M., Steyn, P.S., Creppy, E.E., 1998. Regional selectivity to ochratoxin A, distribution and cytotoxicity in rat brain. Arch. Toxicol. 72: 656-662.

Bendele, A.M., Carlton, W.W., Krogh, P., Lillehoj, E.B., 1985. Ochratoxin A carcinogenesis in the (C57BL/6J X C3H)F 1 mouse. J. Natl. Cancer Inst. 75: 733-742.

Berger, V., Gabriel, A.F., Sergent, T., Trouet, A., Larondelle, Y., Schneider, Y.J., 2003. Interaction of ochratoxin A with human intestinal Caco-2 cells: possible implication of a multidrug resistance-associated protein (MRP2). Toxicol. Lett. 140-141: 465-476.

Bow, D.A., Perry, J.L., Simon, J.D., Pritchard, J.B., 2006. The impact of plasma protein binding on the renal transport of organic anions. J. Pharmacol. Exp. Ther. 316: 349-355.

Breitholtz-Emanuelsson, A., Fuchs, R., Hult, K., 1995. Toxicokinetics of ochratoxin A in rat following intratracheal administration. Nat. Toxins 3: 101-103. 
Breitholtz-Emanuelsson, A., Palminger-Hallen, I., Wohlin, P.O., Oskarsson, A., Hult, K., Olsen, M., 1993. Transfer of ochratoxin A from lactating rats to their offspring: a shortterm study. Nat. Toxins 1: 347-352.

Breitholtz Emanuelsson, A., Fuchs, R., Hult, K., Appelgren, L.E., 1991. Distribution of 14C-ochratoxin A and 14C-ochratoxin B in rats: a comparison based on whole-body autoradiography. IARC Sci Publ 115: 201-203.

Castegnaro, M., Mohr, U., Pfohl-Leszkowicz, A., Esteve, J., Steinmann, J., Tillmann, T., Michelon, J., Bartsch, H., 1998. Sex- and strain-specific induction of renal tumors by ochratoxin A in rats correlates with DNA adduction. Int. J. Cancer 77: 70-75.

Castegnaro, M., Bartsch, H., Bereziat, J.C., Arvela, P., Michelon, J., Broussolle, L., 1989. Polymorphic ochratoxin A hydroxylation in rat strains phenotyped as poor and extensive metabolizers of debrisoquine. Xenobiotica 19: 225-230.

Chandra, P. and Brouwer, K.L.R., 2004. The complexities of hepatic drug transport: current knowledge and emerging concepts. Pharm. Res. 21: 719-735.

Chang, F.C. and Chu, F.S., 1977. The fate of ochratoxin A in rats. Food Cosmet. Toxicol. 15: 199-204.

Dai, J., Park, G., Wright, M., Adams, M., Akman, S., Manderville, R. , 2002. Detection and characterization of a glutathione conjugate of ochratoxin A. Chem. Res. Toxicol. 15: 1581-1588.

Dietrich, D.R., Heussner, A.H., O'Brien, E., 2005. Ochratoxin A: comparative pharmacokinetics and toxicological implications (experimental and domestic animals and humans). Food Addit. Contam. 22 Suppl. 1: 45-52.

Dixit, R., Riviere, J., Krishnan, K., Andersen, M.E., 2003. Toxicokinetics and physiologically based toxicokinetics in toxicology and risk assessment. J. Toxicol. Environ. Health B Crit Rev 6: 1-40.

Domijan, A.M., Rudes, K., Peraica, M., 2005. The effect of ochratoxin A on the concentration of protein carbonyls in rats. Arh. Hig. Rada Toksikol. 56: 311-315.

Eckhardt, U., Schroeder, A., Stieger, B., Hochli, M., Landmann, L., Tynes, R., Meier, P.J., Hagenbuch, B., 1999. Polyspecific substrate uptake by the hepatic organic anion transporter Oatp1 in stably transfected CHO cells. Am. J. Physiol. 276: 1037-1042.

Entwisle, A.C., Jorgensen, K., Williams, A.C., Boenke, A., Farnell, P.J., 1997. An intercomparison of methods for the determination of ochratoxin A in pig kidney. Food Addit. Contam. 14: 223-236. 
EFSA (European Food Safety Authority) (2006) Opinion of the Scientific Panel on contaminants in the food chain on a request from the Commission related to ochratoxin $\mathrm{A}$ in food. Adopted on 4 April 2006. The EFSA Journal 365: 1-56.

Frohlich, A.A., Marquardt, R.R., Bernatsky, A., 1988. Quantitation of ochratoxin A: use of reverse phase thin-layer chromatography for sample cleanup followed by liquid chromatography or direct fluorescence measurement. J. Assoc. Off. Anal. Chem. 71: 949953.

Fuchs R. and Hult K., 1992. Ochratoxin A in blood and its pharmacokinetic properties. Food Chem. Toxicol. 30: 201-204.

Galtier, P., 1991. Pharmacokinetics of ochratoxin A in animals. IARC Sci Publ 115: 187200 .

Galtier, P., Alvinerie, M., Charpenteau, J.L., 1981. The pharmacokinetic profiles of ochratoxin A in pigs, rabbits and chickens. Food. Cosmet. Toxicol. 19: 735-738.

Galtier, P., Charpenteau, J.L., Alvinerie, M., Labouche, C., 1979. The pharmacokinetic profile of ochratoxin A in the rat after oral and intravenous administration. Drug Metab. Dispos. 7: 429-434.

Galtier, P., 1977. Contribution of pharmacokinetic studies to mycotoxicology- ochratoxin A. Veterinary science communications 1:349-358.

Gautier, J., Richoz, J., Welti, D.H., Markovic, J., Gremaud, E., Guengerich, F.P., Turesky, R.J., 2001. Metabolism of ochratoxin A: absence of formation of genotoxic derivatives by human and rat enzymes. Chem. Res. Toxicol. 14: 34-45.

Gillman, I.G., Clark, T.N., Manderville, R.A., 1999. Oxidation of ochratoxin A by an Feporphyrin system: model for enzymatic activation and DNA cleavage. Chem Res. Toxicol. 12: 1066-1076.

Gross-Steinmeyer, K., Weymann, J., Hege, H.G., Metzler, M., 2002. Metabolism and lack of DNA reactivity of the mycotoxin ochratoxin a in cultured rat and human primary hepatocytes. J Agric. Food Chem. 50: 938-945.

Hagelberg, S., Hult, K., Fuchs, R., 1989. Toxicokinetics of ochratoxin A in several species and its plasma-binding properties. J. Appl. Toxicol. 9: 91-96.

Hallen, I.P., Breitholtz-Emanuelsson, A., Hult, K., Olsen, M., Oskarsson, A., 1998. Placental and lactational transfer of ochratoxin A in rats. Nat. Toxins 6: 43-49.

Han, Z., Zhao, Z., Shi, J., Liao, Y., Zhao, Z., Zhang, D., Wu, Y., De Saeger, S., Wu, A., 2013. Combinatorial approach of LC-MS/MS and LC-TOF-MS for uncovering in vivo kinetics and biotransformation of ochratoxin A in rat. J. Chromatogr. B Analyt. Technol. Biomed. Life Sci. 925: 46-53. 
Hult, K., Fuchs, R., Peraica, M., Plestina, R. Ceović, S., 1984. Screening for ochratoxin A in blood by flow injection analysis. J. Appl. Toxicol. 4: 326-329.

Hult, K., Hkby, E., Hgglund, U., Gatenbeck, S., Rutqvist, L., Sellyey, G., 1979. Ochratoxin A in pig blood: method of analysis and use as a tool for feed studies. Appl Environ Microbiol 38: 772-776.

ICH Harmonised Tripartite Guideline, S3A: Note for Guidance on Toxicokinetics: The Assessment of Systemic Exposure in Toxicity Studies, International Conference on Harmonisation of Technical Requirements for Registration of Pharmaceuticals for Human Use (ICH), Geneva, October 1994.

Ito, T., Takahashi, M., Sudo, K., Sugiyama, Y., 2007. Marked strain differences in the pharmacokinetics of an alpha4beta1 integrin antagonist, 4-[1-[3-Chloro-4-[N-(2methylphenyl)-ureido]phenylacetyl]-(4S)-fluoro-(2S)-pyrroldine-2-yl]-methoxybenzoic Acid (D01-4582), in Sprague-Dawley rats are associated with albumin genetic polymorphism. J. Pharmacol. Exp. Ther. 320:124-132.

Jiménez, A., Lopez de Cerain, A-, Gonzalez-Peñas, E., Bello, J. 1999. A High-Performance Liquid-Chromatographic Method for the Determination of Ochratoxin A in Human Plasma, Chromatographia 50: 457-460.

Kane, A., Creppy, E.E., Roth, A., Roschenthaler, R., Dirheimer, G., 1986. Distribution of the $[3 \mathrm{H}]$-label from low doses of radioactive ochratoxin A ingested by rats, and evidence for DNA single-strand breaks caused in liver and kidneys. Arch. Toxicol. 58: 219-224.

Kerkadi, A., Barriault, C., Tuchweber, B., Frohlich, A.A., Marquardt, R.R., Bouchard, G., Yousef, I.M., 1998. Dietary cholestyramine reduces ochratoxin A-induced nephrotoxicity in the rat by decreasing plasma levels and enhancing fecal excretion of the toxin. J. Toxicol. Environ. Health A. 53: 231-250.

Kontaxi, M., Echkardt, U., Hagenbuch, B., Stieger, B., Meier, P.J., Petzinger, E., 1996. Uptake of the mycotoxin ochratoxin A in liver cells occurs via the cloned organic anion transporting polypeptide. J Pharmacol. Exp. Ther. 279: 1507-1513.

Kuiper-Goodman, T. and Scott, P.M., 1989. Risk assessment of the mycotoxin ochratoxin A. Biomed Environ Sci 2: 179-248.

Kumagai, S. 1988. Effects of plasma ochratoxin A and luminal $\mathrm{pH}$ on the jejunal absorption of ochratoxin A in rats. Food Chem. Toxicol. 26: 753-758.

Kumagai S (1985) Ochratoxin A: plasma concentration and excretion into bile and urine in albumin-deficient rats. Food and Chemical Toxicology 23: 941-943.

Kumagai, S. and Aibara, K., 1982. Intestinal absorption and secretion of ochratoxin A in the rat. Toxicol. Appl. Pharmacol. 64: 94-102. 
Li, S., Marquardt, R.R., Frohlich, A.A., 2000. Identification of ochratoxins and some of their metabolites in bile and urine of rats. Food Chem. Toxicol. 38: 141-152.

Li, S., Marquardt, R.R., Frohlich, A.A., Vitti, T.G., Crow, G., 1997. Pharmacokinetics of ochratoxin A and its metabolites in rats. Toxicol. Appl. Pharmacol. 145: 82-90.

Madhyastha, M.S., Frohlich, A.A., Marquardt, R.R., 1992. Effect of dietary cholestyramine on the elimination pattern of ochratoxin A in rats. Food Chem. Toxicol. 30: 709-714.

Mally, A. and Dekant, W., 2009. Mycotoxins and the kidney: modes of action for renal tumor formation by ochratoxin A in rodents. Mol. Nut. Food Res. 53: 467-478.

Mally, A., Keim-Heusler, H., Amberg, A., Kurz, M., Zepnik, H., Mantle, P., Vlkel, W., Hard, G., Dekant, W., 2005. Biotransformation and nephrotoxicity of ochratoxin B in rats. Toxicol. Appl. Pharmacol. 206: 43-53.

Mally, A., Zepnik, H., Wanek, P., Eder, E., Dingley, K., Ihmels, H., Volkel, W. , Dekant, W., 2004. Ochratoxin A: lack of formation of covalent DNA adducts. Chem. Res. Toxicol. 17: 234-242.

Mantle, P. and Nagy, J., 2008. Binding of a Ochratoxin A to a Urinary Globulin: A New Concept to Account for Gender Difference in Rat Nephrocarcinogenic Responses. Int. J. Mol. Sci 9: 719-735.

Mantle, P.G., 2008. Interpretation of the pharmacokinetics of ochratoxin A in blood plasma of rats, during and after acute or chronic ingestion. Food Chem. Toxicol. 46: 1808-1816.

Mantle, P., Kulinskaya, E., Nestler, S., 2005. Renal tumourigenesis in male rats in response to chronic dietary ochratoxin A. Food Addit. Contam. 22 Suppl 1: 58-64.

Maresca, M, Mahfoud, R., Pfohl-Leszkowicz, A., Fantini, J., 2001. The mycotoxin ochratoxin A alters intestinal barrier and absorption functions but has no effect on chloride secretion. Toxicol. Appl. Pharmacol. 176: 54-63.

Miljkovic, A., Pfohl-Leszkowicz, A., Dobrota, M. Mantle, P.G., 2003. Comparative responses to mode of oral administration and dose of ochratoxin A or nephrotoxic extract of Penicillium polonicum in rats. Exp. Toxicol. Pathol. 54: 305-312.

Muñoz, K., Blaszkewicz, M., Degen, G., 2010. Simultaneous analysis of ochratoxin A and its major metabolite ochratoxin alpha in plasma and urine for an advanced biomonitoring of the mycotoxin. J. Chromatogr. B Analyt. Technol. 878 :2623-2629.

National Toxicology Program (NTP) (1989) Toxicology and carcinogenesis studies of ochratoxin A (CAS No 303-47-9) in F344/N rats (gavage studies). Nat 1 Toxicol Program Tech Rep Ser 358: 1-142. 
Obrecht-Pflumio, S., Grosse, Y., Pfohl-Leszkowicz, A., Dirheimer, G., 1996. Protection by indomethacin and aspirin against genotoxicity of ochratoxin A, particularly in the urinary bladder and kidney. Arch. Toxicol. 70: 244-248.

O'Brien, E. and Dietrich, D.R., 2005. Ochratoxin A: the continuing enigma. Crit. Rev. Toxicol. 35: 33-60.

OECD guideline for the testing of chemicals 417. 2010. Toxic okinetic s.PfohlLeszkowicz, A., Manderville, R.A., 2012. An update on direct genotoxicity as a molecular mechanism of ochratoxin a carcinogenicity Chem. Res. Toxicol. 25: 252-262.

Pfohl-Leszkowicz, A. and Manderville, R.A., 2007. Ochratoxin A: An overview on toxicity and carcinogenicity in animals and humans. Mol. Nutr. Food Res. 51: 61-99.

Pfohl-Leszkowicz, A. and Castegnaro, M., 2005. Further arguments in favour of direct covalent binding of Ochratoxin A (OTA) after metabolic biotransformation. Food Addit. Contam. 22 Suppl 1: 75-87.

Pfohl-Leszkowicz, A., Pinelli, E., Bartsch, H., Mohr, U., Castegnaro, M., 1998. Sex- and strain-specific expression of cytochrome P450s in ochratoxin A-induced genotoxicity and carcinogenicity in rats. Mol. Carcinog. 23: 76-85.

Rached,, E., Hard, G.C., Blumbach, K., Weber, K., Draheim, R., Lutz, W.K., Ozden, S., Steger, U., Dekant, W., Mally, A., 2007. Ochratoxin A: 13-week oral toxicity and cell proliferation in male F344/n rats. Toxicol. Sci. 97: 288-298.

Rasonyi, T., Schlatter, J., Dietrich, D.R., 1999. The role of alpha2u-globulin in ochratoxin A induced renal toxicity and tumors in F344 rats. Toxicol. Lett. 104: 83-92.

Ringot, D., Chango, A., Schneider, Y.J., Larondelle, Y., 2006. Toxicokinetics and toxicodynamics of ochratoxin A, an update. Chem. Biol. Interact. 159: 18-46.

Rodgers, I.S. and Baetcke, K.P., 1993. Interpretation of male rat renal tubule tumors. Environ. Health Perspect. 101 Suppl 6: 45-52.

Roth, A., Chakor, K., Creppy, E.E., Kane, A., Roschenthaler, R., Dirheimer, G., 1988. Evidence for an enterohepatic circulation of ochratoxin A in mice. Toxicology 48: 293-308.

Russel, F.G.M., Masereeuw, R., van Aubel Remon, A.M.H., 2002. Molecular aspects of renal anionic drug transport. Annu, Rev Physiol 64: 563-594.

Sakai, N. and Ishizuka, M., 2009. Impact of rat P450 genetic polymorphism on diazepam metabolism. Expert Opin. Drug Metab. Toxicol. 5: 1421-1433. 
Sauvant, C., Silbernagl, S., Gekle, M., 1998. Exposure to ochratoxin A impairs organic anion transport in proximal-tubule-derived opossum kidney cells. J. Pharmacol. Exp. Ther. 287: $13-20$.

Schaut, A., De Saeger, S., Sergent, T., Schneider, Y., Larondelle, Y., Pussemier, L., Blank, R., Van Peteghem, C., 2008. Liquid chromatographic methods for biotransformation studies of ochratoxin A. Biomed. Chromatogr. 22: 1013-1020.

Schrickx, J., Lektarau, Y., Fink-Gremmels, J., 2006. Ochratoxin A secretion by ATPdependent membrane transporters in Caco-2 cells. Arch. Toxicol. 80: 243-249.

Schwerdt, G., Bauer, K., Gekle, M., Silbernagl, S., 1996. Accumulation of ochratoxin A in rat kidney in vivo and in cultivated renal epithelial cells in vitro. Toxicology 114: 177-185.

Stoev, S.D., Paskalev, M., MacDonald, S., Mantle, P.G., 2002. Experimental one year ochratoxin A toxicosis in pigs. Exp. Toxicol. Pathol. 53 :481-487.

Stojkovic, R., Hult, K., Gamulin, S., Plestina, R., 1984. High affinity binding of ochratoxin A to plasma constituents. Biochem. Int. 9: 33-38.

Storen, O., Holm, H., Stormer, F.C., 1982. Metabolism of ochratoxin A by rats. Appl. Environ. Microbiol. 44: 785-789.

Subramanian, S., Balasubramanian, N., William, S., Govindasamy, S., 1991. In vivo absorption of $14 \mathrm{C}$-glucose and $14 \mathrm{C}$-glycine by the rat intestine during ochratoxin A toxicosis. Biochem. Int. 23: 655-661.

Suzuki, S., Satoh, T., Yamazaki, M., 1977. The pharmacokinetics of ochratoxin A in rats. Jpn. J. Pharmacol. 27: 735-744.

Swenberg, J.A., 1993. Alpha 2u-globulin nephropathy: review of the cellular and molecular mechanisms involved and their implications for human risk assessment. Environ. Health Perspect. 101 Suppl. 6: 39-44.

Takeuchi, A., Masuda, S., Saito, H., Abe, T., Inui, K., 2001. Multispecific substrate recognition of kidney-specific organic anion transporters OAT-K1 and OAT-K2. J. Pharmacol. Exp. Ther. 299: 261-267.

Tozlovanu, M., Canadas, D., Pfohl-Leszkowicz, A., Frenette, C., Paugh, R.J., Manderville, R.A., 2012. Glutathione conjugates of ochratoxin A as biomarkers of exposure. Arh. Hig. Rada Toksikol. 63:417-427.

Tsuda, M., Sekine, T., Takeda, M., Cha, S.H., Kanai, Y., Kimura, M., Endou, H., 1999. Transport of ochratoxin A by renal multispecific organic anion transporter 1. J. Pharmacol. Exp. Ther. 289: 1301-1305. 
Valenta, H., 1998. Chromatographic methods for the determination of ochratoxin A in animal and human tissues and fluids. J. Chromatogr. A 815: 75-92.

Vettorazzi, A., de Trocóniz, I.F., González-Peñas, E., Arbillaga, L., Corcuera, L.A., Gil, A.G., de Cerain, A.L., 2011. Kidney and liver distribution of ochratoxin A in male and female F344 rats. Food Chem. Toxicol. 49:1935-1942.

Vettorazzi, A., Trocóniz, I.F., Gonzalez-Peñas, E., Corcuera, L.A., Arbillaga, L., Gil, A.G., Nagy, J.M., Mantle, P.G., de Cerain, A.L., 2010. Effects of fasting and gender on ochratoxin A toxicokinetics in F344 rats. Food Chem. Toxicol. 48:3159-3166.

Vettorazzi, A., Gonzalez-Peñas, E., Troconiz, I.F., Arbillaga, L., Corcuera, L.A., Gil, A.G., Lopez de Cerain, A.L., 2009. A different kinetic profile of ochratoxin A in mature male rats. Food Chem. Toxicol. 47: 1921-1927.

Vettorazzi, A., Gonzalez-Peñas, E., Arbillaga, L., Corcuera, L.A., Lopez de Cerain, A., 2008. Simple high-performance liquid chromatography-fluorescence detection method for plasma, kidney and liver of rat as a tool for toxicology studies. J. Chromatogr. A 1215: 100-106.

Wang, L., Sweet, D.H., 2013. Renal organic anion transporters (SLC22 family): expression, regulation, roles in toxicity, and impact on injury and disease . AAPS J. 15:5369.

WHO (World Health Organisation) (2008) Safety evaluation of certain food additives and contaminants. WHO Food Additives Series, No. 59.

WHO (World Health Organisation) (2001) Safety evaluation of certain mycotoxins in food. WHO Food Additives Series, No. 47.

Wu, Q., Dohnal, V., Huang, L., Kuča, K., Wang, X., Chen, G., Yuan, Z., 2011. Metabolic pathways of ochratoxin A. Curr. Drug Metab. 12:1-10. Review.

Xiao, H., Madhyastha, S., Marquardt, R.R., Li, S, Vodela, J.K., Frohlich, A.A. Kemppainen, B.W. ,(1996)a Toxicity of ochratoxin A, its opened lactone form and several of its analogs: structure-activity relationships. Toxicol. Appl. Pharmacol. 137: 182-192.

Xiao, H., Marquardt, R.R., Abramson, D., Frohlich, A.A., (1996)b. Metabolites of ochratoxins in rat urine and in a culture of Aspergillus ochraceus. Appl. Environ. Microbiol. 62: 648-655.

Zepnik, H., Völkel, W., Dekant, W., 2003. Toxicokinetics of the mycotoxin ochratoxin A in F 344 rats after oral administration. Toxicol. Appl. Pharmacol. 192: 36-44.

Zepnik, H., Pahler, A., Schauer, U., Dekant, W., 2001. Ochratoxin A-induced tumor formation: is there a role of reactive ochratoxin A metabolites? Toxicol. Sci. 59: 59-67. 
Zlender, V., Breljak, D., Ljubojevic, M., Flajs, D., Balen, D., Brzica, H., Domijan, A.M., Peraica, M., Fuchs, R., Anzai, N., Sabolic, I., 2009. Low doses of ochratoxin A upregulate the protein expression of organic anion transporters Oat1, Oat2, Oat3 and Oat5 in rat kidney cortex. Toxicol. Appl. Pharmacol. 239: 284-296.

Figures:

Figure. 1. Ochratoxin A metabolites detected in different biological matrices of rats. (Figure modified from Pfohl-Leszkowicz and Manderville, 2007). Biological matrices in which metabolites were detected appear indicated in parenthesis. Only metabolites detected by HPLC-FLD or HPLC-MS are shown.

References: ${ }^{1}$ (Galtier et al., 1979; Storen et al., 1982; Suzuki et al., 1977; Xiao et al., 1996b ; Zepnik et al., 2003 ; Mally et al., 2004 ; Tozlavanu et al., 2012); ${ }^{2}$ (Storen et al., 1982; Xiao et al., 1996b);

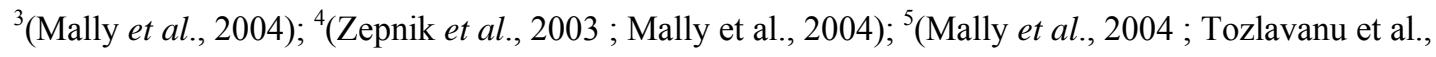
2012); ${ }^{6}$ (Li et al., 2000); ${ }^{7}$ (Han et al., 2013). 